\title{
Dynamic, mating-induced gene expression changes in female head and brain tissues of Drosophila melanogaster
}

\author{
Justin E Dalton ${ }^{\dagger}$, Tanvi S Kacheria ${ }^{\dagger}$, Simon RV Knott ${ }^{\dagger}$, Matthew S Lebo ${ }^{\dagger}$, Allison Nishitani ${ }^{\dagger}$, Laura E Sanders ${ }^{\dagger}$, \\ Emma J Stirling ${ }^{\dagger}$, Ari Winbush ${ }^{\dagger}$, Michelle N Arbeitman ${ }^{*}$
}

\begin{abstract}
Background: Drosophila melanogaster females show changes in behavior and physiology after mating that are thought to maximize the number of progeny resulting from the most recent copulation. Sperm and seminal fluid proteins induce post-mating changes in females, however, very little is known about the resulting gene expression changes in female head and central nervous system tissues that contribute to the post-mating response.

Results: We determined the temporal gene expression changes in female head tissues 0-2, 24, 48 and 72 hours after mating. Females from each time point had a unique post-mating gene expression response, with 72 hours post-mating having the largest number of genes with significant changes in expression. At most time points, genes expressed in the head fat body that encode products involved in metabolism showed a marked change in expression. Additional analysis of gene expression changes in dissected brain tissues 24 hours post-mating revealed changes in transcript abundance of many genes, notably, the reduced transcript abundance of genes that encode ion channels.
\end{abstract}

Conclusions: Substantial changes occur in the regulation of many genes in female head tissues after mating, which might underlie aspects of the female post-mating response. These results provide new insights into the physiological and metabolic changes that accompany changes in female behaviors.

\section{Background}

Successful reproduction in Drosophila melanogaster requires the interplay of behavioral repertoires performed by males and females, which include male courtship, female receptivity, copulation, and female post-mating responses. Females are biochemically poised to respond to sperm, proteins, and other molecules transferred during copulation, which induce the postmating response. This response, which lasts about a week, includes reduced receptivity to mating, metabolic changes, increased immunity and the physiological changes that accompany sperm storage, fertilization and egg-laying [reviewed in [1,2]].

\footnotetext{
* Correspondence: arbeitma@usc.edu

+ Contributed equally

Section of Molecular and Computational Biology, Department of Biological Sciences, University of Southern California, Los Angeles, California 90089,
} USA

(c) 2010 Dalton et al; licensee BioMed Central Ltd. This is an Open Access article distributed under the terms of the Creative Commons Attribution License (http://creativecommons.org/licenses/by/2.0), which permits unrestricted use, distribution, and reproduction in any medium, provided the original work is properly cited.
Accessory gland proteins (Acps), which are synthesized in the male accessory gland and transferred to females in the seminal fluid during copulation, act together to induce the post-mating response (reviewed in [1-3]). Of the Acps identified, sex peptide (SP) appears to be one of the primary triggers of the postmating response. Injection of biochemically purified SP or ectopic expression of the gene that encodes SP in virgin females induces increased egg laying and reduced receptivity to mating $[4,5]$. Furthermore, females mated to males lacking SP show a diminished post-mating response, as they are more receptive to mating and lay fewer eggs than females mated to wild type males [6,7]. $\mathrm{SP}$ enters the female reproductive tract during copulation. A fraction of SP immediately circulates systemically by entering into the hemolymph [8], whereas another fraction remains bound to the tail of sperm and is thought to be gradually released into the hemolymph by cleavage $[9,10]$. SP binds the sex peptide receptor (SPR),
C Biomed Central 
a G protein-coupled receptor [11], which is expressed in the female reproductive organs and nervous system. SPR is required in a subset of sensory neurons in the reproductive tract to elicit the post-mating response [12,13]. Additionally, it has been shown that after mating females increase their food intake and have a preference for food with yeast, a source of proteins, which is thought to facilitate increased egg production. This change in food preference and intake requires SPR and receipt of SP [14-16].

Females that receive seminal fluid proteins, but no sperm, have a short-term post-mating response that lasts about a day, with increased egg production and decreased receptivity to mating. Long-term post-mating changes, which last about a week, require receipt of both sperm and seminal fluid proteins, with SP playing a critical role $[9,10]$. The slow release of SP that is bound to sperm tails is thought to elicit the long-term female post-mating changes. Other Acps contribute to the post-mating response in different ways including increasing ovulation rate (Acp26Aa, also called Ovulin; [17]) and sperm storage (Acp36DE; [18]). Additionally, CG33943 is required for full stimulation of egg laying, and four additional Acps (CG1652, CG1656, CG17575, CG9997) are required for sustained egg laying, release of sperm from storage organs, and reduced receptivity to courtship [10]. The functions of some 100 other Acps are largely unknown.

Despite having knowledge regarding the functions of seminal fluid proteins that contribute to the post-mating response, little is known about the downstream effector genes that mediate the post-mating response in females, especially in tissues outside of the reproductive tract, such as head and central nervous system (CNS) tissues. Microarray studies have determined the global changes in transcription observed after mating by comparing gene expression between virgin and mated females in whole animals [19-21] and in dissected female reproductive tract tissues [22]. It is clear that there are tissue-specific, post-mating gene expression responses [compare $[19,22]$; however, it is unlikely that changes in gene expression in head and brain tissues would have been detected by the previous whole animal studies given the sensitivity of the microarray assay [see [23,24]. Finally, while the post-mating response lasts for about a week, previous genomic studies only examined time points within 24 hours of copulation. Important, unanswered questions remain, including the identification of tissue-specific gene expression changes that occur in head and brain tissues post-mating and understanding how the post-mating response is maintained long-term.

Here, we present the results of a time-course study examining gene expression in head and dissected brain tissues of female flies after mating. Genes whose products function in metabolism show marked changes in expression levels in the head post-mating. A large proportion of these genes are expressed in the head fat body, a tissue that is similar to mammalian adipose tissue. The fat body can influence tissues at a distance through the release of factors into the hemolymph [25], as well as respond to energy needs by altering the storage of energy reserves, such as glycogens, lipids, and proteins [26]. We also identified genes that are expressed in the brain whose transcript abundance changes post-mating, including at least five genes that encode products with ion channel activity, suggesting that changes in neurophysiology mediate aspects of the post-mating response. Comparisons of the genes identified in this study with those previously identified in studies examining gene expression changes post-mating in whole animal or internal reproductive tissues revealed little overlap, highlighting that different tissues have a unique post-mating response at the level of gene expression.

\section{Results}

We performed gene expression analyses to identify genes differentially expressed between virgin and mated females, in adult head and brain tissues. RNA was derived from age-matched, adult head tissues from virgin and mated females at 0-2, 24, 48, and 72 hours post-mating, and from brain tissues at 24 hours postmating. For each time point, two-color, glass-slide microarray experiments were performed, using four independent biological replicates [for array platform [27]. All females were five days old when mated and raised under constant environmental conditions, and therefore, were expected to show minimal variation in gene expression levels [28]. Given this, most gene expression differences observed in these experiments are expected to be due to the post-mating response, rather than due to changes in gene expression in virgin female animals. We refer to genes with higher expression in mated females than in age-matched virgins as induced or up-regulated, and those with lower expression in mated females as repressed or down-regulated.

At the four time points examined, differences in the numbers of genes that showed significant differential expression between mated and virgin female head tissues were observed [0-2 (237 genes), 24 (326 genes), 48 (449 genes), and 72 (545 genes) hours post-mating, $P<0.05$ ], with later times showing greater differences (Table 1). These results are distinct from those obtained from experiments that analyzed post-mating gene expression changes in whole adult females, in which thousands of genes showed significant expression differences early, at 1-3 hours post-mating, whereas only hundreds of genes were differentially expressed at each later time point, 
Table 1 The number of genes with significant differences in expression in head tissues post-mating at four time points $(P<0.05)$

\begin{tabular}{ll}
\hline Time point & Number of genes \\
\hline $\begin{array}{l}\text { 0-2 hours post-mating } \\
\text { lower in mated females } \\
\text { higher in mated females }\end{array}$ & 93 \\
$\frac{24 \text { hours post-mating }}{\text { lower in mated females }}$ & 144 \\
higher in mated females & 208 \\
& 118 \\
$\frac{48 \text { hours post-mating }}{\text { lower in mated females }}$ & \\
higher in mated females & 283 \\
72 hours post-mating & 166 \\
\hline lower in mated females & \\
higher in mated females & 316 \\
\hline
\end{tabular}

including 6, 12, and 24 hours after mating [20]. The postmating changes in gene expression in head tissues were also distinct from those identified in the female reproductive tract tissues, in which the most differentially expressed genes were identified at 6 hours post-mating, with fewer identified at an earlier and later time point, 3 hours and 24 hours post-mating, respectively [22].

The difference in kinetics of the transcript changes likely reflects the diverse causes of gene expression changes in the tissues examined. Multiple factors might contribute to expression changes, including social and physical interactions [29], responses to individual seminal fluid components and sperm [20], and responses to fertilization and egg-laying [30]. Additionally, each tissue-type examined likely responds in a tissue- and temporal-specific manner. Given the experimental design, we are unable to distinguish between these different influences on gene expression changes.

\section{Biological processes that underlie the post-mating response in head tissues}

To gain insight into the types of biological processes that are responsive to mating in adult head tissues, we focused our analysis on the top 100 genes which showed significantly altered expression, based on false-discovery rank (FDR), from each time point (Additional file 1). Collectively, there are 309 unique genes from all four time points among the top 100 FDR ranked genes (genes present in multiple lists are counted once; Additional file 1). The genes identified at the 24 and 48 hour post-mating time points show the largest amount of overlap (50 genes overall; six up-regulated and 44 down-regulated; Figure 1). Not surprisingly, the genes from 0-2 and 72 hours stages show the least amount of overlap (two genes; one up-regulated and one that is significantly different at both time points, but regulated in opposite directions; Figure 1).

Only one gene, CG8147, is among the top 100 FDR ranked genes at all four stages; CG8147 encodes a product with predicted alkaline phosphatase activity [31]. CG8147 is significantly induced at all post-mating stages in head and brain tissues examined, except at the 0-2 hour stage in head tissues, where it is significantly repressed. In our previous study, we found that CG8147 shows significant female-biased expression in head tissues [24], perhaps due to up-regulated expression in females post-mating. Others studies show that CG8147

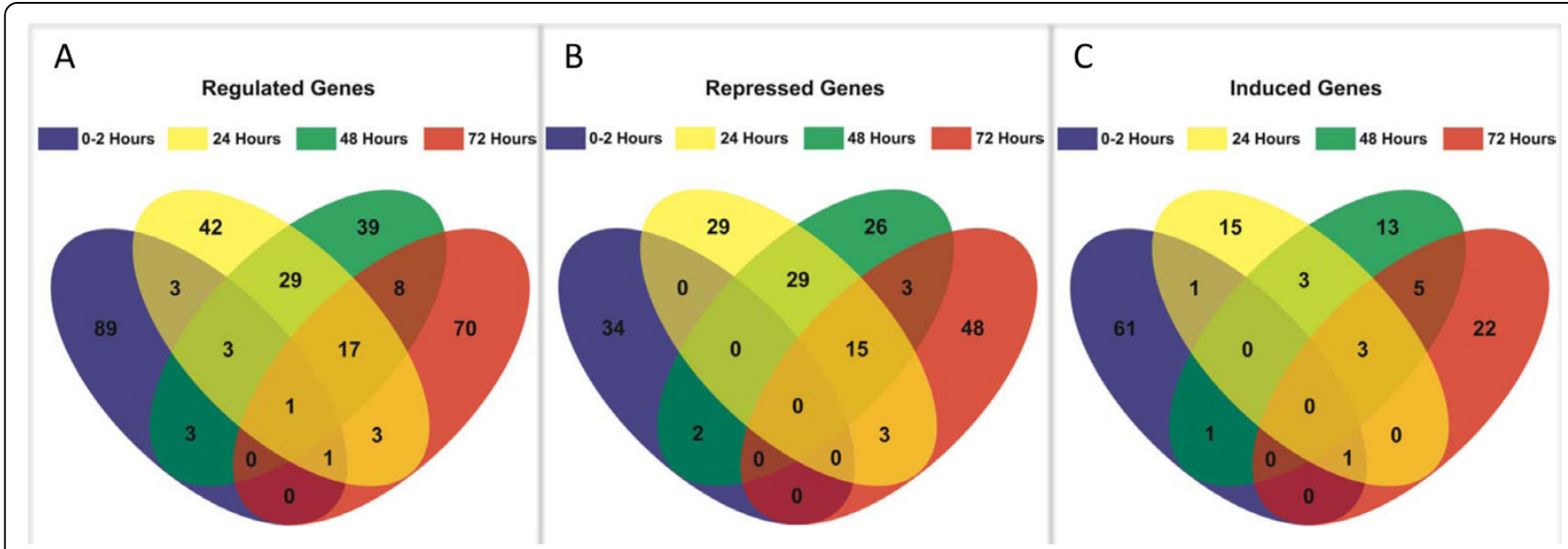

Figure 1 Venn Diagrams showing the number of genes with significant differential expression that overlap among the four postmating time points analyzed. The top 100 ranked FDR genes identified as differentially expressed 0-2 (blue), 24 (yellow), 48 (green) or 72 (red) hours post-mating were used in the analyses. The number of all genes that overlap (A), only the genes that are down-regulated post-mating (B), and only the genes that are up-regulated post-mating (C) among the four time points is shown and indicated by the overlapping colors. 
is highly repressed when flies are starved and suggest that there is a trade-off between nutrient status and reproductive status [32].

Examination of the Gene Ontology (GO) functional groups in the list of 309 genes allows for a global view of post-mating gene expression changes and reveals that changes in transcript abundance of genes that function in metabolism accompany the physiological changes that occur post-mating in head tissues (Table 2 and Additional file 1 identified using Flymine [33]). Nearly all of the 75 significant GO functional categories identified, including those most significantly overrepresented, contain genes that encode products involved in metabolic processes, including metabolism of amino acids, carbohydrates, and nucleotides (Table 2). We were not able to identify metabolic pathways for which all, or most, genes encoding enzymes in the pathway show expression differences in this study. This may be because each gene in the pathway is not regulated at the level of gene expression, or alternatively, because expression differences for these genes do not fall within the top 100 FDR ranked genes.

\begin{tabular}{|c|c|}
\hline \multicolumn{2}{|l|}{ Most significant functional categories identified } \\
\hline GO Functional Category & $P$ value \\
\hline small molecule metabolic process & $2.02 \mathrm{E}-10$ \\
\hline organic acid metabolic process & $5.58 \mathrm{E}-10$ \\
\hline carboxylic acid metabolic process & $5.58 \mathrm{E}-10$ \\
\hline oxoacid metabolic process & $5.58 \mathrm{E}-10$ \\
\hline cellular ketone metabolic process & 3.16E-09 \\
\hline cellular amine metabolic process & 4.44E-08 \\
\hline cellular amino acid metabolic process & 1.39E-07 \\
\hline oxidation reduction & 3.83E-07 \\
\hline cellular amino acid and derivative metabolic process & 5.36E-07 \\
\hline alcohol metabolic process & 1.16E-06 \\
\hline pentose-phosphate shunt & 2.10E-05 \\
\hline NADP metabolic process & 2.10E-05 \\
\hline $\mathrm{NADPH}$ regeneration & 2.10E-05 \\
\hline amine biosynthetic process & 3.29E-05 \\
\hline alcohol catabolic process & 4.58E-05 \\
\hline amine metabolic process & 5.95E-05 \\
\hline coenzyme metabolic process & 1.12E-04 \\
\hline serine family amino acid metabolic process & 1.14E-04 \\
\hline glycine metabolic process & 1.18E-04 \\
\hline nicotinamide metabolic process & 1.19E-04 \\
\hline alkaloid metabolic process & 1.19E-04 \\
\hline nicotinamide nucleotide metabolic process & 1.19E-04 \\
\hline cellular amino acid biosynthetic process & $1.38 \mathrm{E}-04$ \\
\hline pyridine nucleotide metabolic process & 1.83E-04 \\
\hline small molecule catabolic process & 2.01E-04 \\
\hline
\end{tabular}

Gene expression in head tissues $\mathbf{0 - 2}$ hours post-mating Of the top 100 FDR ranked genes, 36 genes are repressed and 64 genes are induced $0-2$ hours postmating (Additional file 1). Of these 100 genes, eight repressed and 18 induced genes show at least two-fold differences in expression (Table 3).

The rapidity of the gene expression response suggests that Acps, or other small molecules contained in the seminal fluid, rapidly circulate in the female's hemolymph and influence gene expression in the head. The mode of signaling may be either direct, as SP has been found associated with brain tissues [34], or indirect, by influencing the physiology of other tissues, like the internal reproductive tissues and associated neurons, which may produce signaling molecules that influence gene expression at distant sites in the body [30]. Given how recently mating had occurred before collecting animals at this time point, the gene expression changes could also be due to social interactions, as it has been shown that males that have courtship interactions, but do not mate, have immediate gene expression changes [29].

\section{Genes with lower expression in females $\mathbf{0 - 2}$ hours after mating}

Genes that are repressed 0-2 hours after mating include those that encode products involved in stress, immunity, or detoxification (Heat shock protein 26, Heat shock protein 68, Heat shock gene 67Bb, relish, Glutathione S transferase E1; Table 4 and Additional file 2 for biological enrichment). Expression of these genes might normally be high in virgin females, with part of the early post-mating response being a reduction of their expression. This result is in contrast to post-mating gene expression changes in whole animals where genes that encode products involved in immunity were up-regulated postmating [20]. Additionally, SP induces the innate immune response [35]. One possible explanation for this discrepancy is that exposure to $\mathrm{CO}_{2}$, the anesthetic used here, induces a stress response. While our experimental design controlled for this by exposing both mated and unmated females to $\mathrm{CO}_{2}$, it is possible that a small increase in the exposure time of virgin females as compared to mated females could account for the apparent repression of genes with products involved in immunity. At all other time points examined flies had at least 24 hours to recover from $\mathrm{CO}_{2}$ exposure and work in our lab has demonstrated that $\mathrm{CO}_{2}$ exposure has no effect on gene expression after 8 hours (unpublished data).

\section{Genes with higher expression in females 0-2 hours after mating}

Dopamine is required for female receptivity to courtship [36] and accordingly, genes that are up-regulated in response to mating include those involved in dopamine 
Table 3 The five genes with the most substantial differences in expression that are induced and repressed post-mating at each time point

\begin{tabular}{|c|c|c|c|c|}
\hline \multicolumn{5}{|c|}{ Higher in females $0-2$ hours post-mating } \\
\hline Flybase & symbol & Fold-change & FDR & FDR rank \\
\hline FBgn0052350 & CG32350 & 2.67 & $1.54 \mathrm{E}-03$ & 13 \\
\hline FBgn0004396 & CrebA & 2.77 & 7.49E-04 & 10 \\
\hline FBgn0086265 & Fcp26Aa & 4.83 & $1.31 \mathrm{E}-02$ & 65 \\
\hline FBgn0035741 & BBS1 & 5.44 & $1.72 \mathrm{E}-04$ & 5 \\
\hline FBgn0033521 & CG12896 & 2.56 & $1.15 \mathrm{E}-05$ & 1 \\
\hline \multicolumn{5}{|c|}{$\underline{\text { Lower in females } 0-2 \text { hours post-mating }}$} \\
\hline Flybase & symbol & Fold-change & FDR & FDR rank \\
\hline FBgn0001225 & Hsp26 & 11.98 & $1.50 \mathrm{E}-04$ & 4 \\
\hline FBgn0035752 & CG8616 & 10.91 & 3.19E-05 & 2 \\
\hline FBgn0001230 & Hsp68 & 7.08 & $3.78 \mathrm{E}-05$ & 3 \\
\hline FBgn0001228 & Hsp67Bb & 3.22 & 4.25E-04 & 6 \\
\hline FBgn0014018 & Rel & 2.55 & $1.57 \mathrm{E}-02$ & 85 \\
\hline \multicolumn{5}{|c|}{ Higher in females 24 hours post-mating } \\
\hline Flybase & symbol & Fold-change & FDR & FDR rank \\
\hline FBgn0036381 & CG8745 & 2.56 & 8.35E-03 & 80 \\
\hline FBgn0003250 & Rh4 & 2.67 & $3.39 \mathrm{E}-03$ & 42 \\
\hline FBgn0040074 & retinin & 3.13 & $5.14 \mathrm{E}-03$ & 62 \\
\hline FBgn0040609 & CG3348 & 3.56 & $3.00 \mathrm{E}-04$ & 7 \\
\hline FBgn0043791 & CG8147 & 4.39 & $8.76 \mathrm{E}-03$ & 84 \\
\hline \multicolumn{5}{|c|}{ Lower in females 24 hours post-mating } \\
\hline Flybase & symbol & Fold-change & FDR & FDR rank \\
\hline FBgn0002565 & Lsp2 & 18.47 & 4.53E-06 & 1 \\
\hline FBgn0002563 & Lspibeta & 12.14 & $1.00 \mathrm{E}-04$ & 2 \\
\hline FBgn0039330 & CG11909 & 9.05 & $1.26 \mathrm{E}-04$ & 3 \\
\hline FBgn0039685 & Obp99b & 5.44 & $1.31 \mathrm{E}-03$ & 26 \\
\hline FBgn0040349 & CG3699 & 5.32 & 8.31E-04 & 19 \\
\hline \multicolumn{5}{|c|}{ Higher in females 48 hours post-mating } \\
\hline Flybase & symbol & Fold-change & FDR & FDR rank \\
\hline FBgn0040502 & CG8343 & 3.52 & $9.05 \mathrm{E}-06$ & 10 \\
\hline FBgn0039703 & CG7829 & 3.66 & $1.51 \mathrm{E}-05$ & 15 \\
\hline FBgn0085353 & CG34324 & 5.32 & $3.98 \mathrm{E}-05$ & 24 \\
\hline FBgn0030773 & CG9676 & 6.70 & 5.34E-07 & 4 \\
\hline FBgn0043791 & CG8147 & 6.96 & 4.31E-04 & 47 \\
\hline \multicolumn{5}{|c|}{ Lower in females 48 hours post-mating } \\
\hline Flybase & symbol & Fold-change & FDR & FDR rank \\
\hline FBgn0002565 & Lsp2 & 16.60 & $9.39 \mathrm{E}-08$ & 1 \\
\hline FBgn0002563 & Lspibeta & 9.10 & $3.69 \mathrm{E}-07$ & 2 \\
\hline FBgn0039685 & Obp99b & 9.09 & $1.93 \mathrm{E}-05$ & 17 \\
\hline FBgn0033830 & CG10814 & 6.59 & $1.66 \mathrm{E}-05$ & 16 \\
\hline FBgn0030073 & CG10962 & 5.41 & $2.73 \mathrm{E}-05$ & 20 \\
\hline \multicolumn{5}{|c|}{ Higher in females 72 hours post-mating } \\
\hline Flybase & symbol & Fold-change & FDR & FDR rank \\
\hline FBgn0037612 & CG8112 & 2.17 & 4.10E-04 & 20 \\
\hline FBgn0052523 & CG32523 & 2.35 & $7.54 \mathrm{E}-05$ & 7 \\
\hline FBgn0043791 & CG8147 & 3.07 & $2.01 \mathrm{E}-03$ & 67 \\
\hline FBgn0000165 & $B C$ & 3.52 & $8.10 \mathrm{E}-05$ & 8 \\
\hline FBgn0040609 & CG3348 & 5.84 & $3.01 \mathrm{E}-06$ & 2 \\
\hline
\end{tabular}

Table 3: The five genes with the most substantial differences in expression that are induced and repressed postmating at each time point (Continued)

\begin{tabular}{lllll}
\hline \multicolumn{2}{l}{ Lower in females 72 hours post-mating } & & \\
\hline Flybase & symbol & Fold-change & FDR & FDR rank \\
FBgn0033830 & CG10814 & 7.87 & $2.67 \mathrm{E}-06$ & 1 \\
FBgn0039685 & Obp99b & 5.65 & $3.98 \mathrm{E}-06$ & 3 \\
FBgn0039298 & to & 4.18 & $1.01 \mathrm{E}-05$ & 5 \\
FBgn0031432 & Cyp309a1 & 3.27 & $9.35 \mathrm{E}-05$ & 10 \\
FBgn0029158 & Las & 3.11 & $8.46 \mathrm{E}-05$ & 9 \\
\hline
\end{tabular}

metabolism (Table 4 and Additional File 2). pale, which encodes a tyrosine hydroxylase important for the production of dopamine, was up-regulated immediately after mating (1.65 fold-change [FC]; FDR rank 25). This is consistent with dopamine playing a role in female receptivity, as the 0-2 hour time point includes females that were recently receptive to courtship. Since acetylation is thought to lead to dopamine inactivation, the upregulation of dopamine $\mathrm{N}$-acetyltransferase (1.43 FC; FDR rank 42), which converts dopamine to $\mathrm{N}$-acetyl dopamine $[37,38]$, provides a mechanism to achieve a subsequent reduction in dopamine activity.

female-independent-of-transformer (fit) showed increased expression in 0-2 hour post-mated head tissues (1.60 FC; FDR rank 15), whereas fit showed decreased expression at later times. Other studies examining the post-mating gene expression response in whole females also found significantly increased fit expression at several stages $[19,21]$, consistent with fit playing a key, but unknown role in the post-mating response in many tissues. The biochemical function of Fit is not known, but fit is highly expressed in the fat body [39]. Furthermore, fit has also been shown to be down-regulated in response to starvation [32] and upregulated in males in response to courtship interactions [29]. Taken together, fit appears to mediate or respond to reproductive and nutrient status in both males and females.

Additional genes that are up-regulated early in response to mating include diminutive, which encodes a transcriptional regulator important for animal growth and development ( $d m$, also known as $d M y c ; 1.74 \mathrm{FC}$; FDR rank 52; [40]). Also, one fruitless isoform (microarray probe for the A DNA binding domain) is upregulated in response to mating (2.04 FC; FDR rank 21). fruitless isoforms common to both sexes, including those with the A DNA binding domain, are expressed in the adult brain and may play a role in neural patterning $[41,42]$. In addition, the gene list includes short neuropeptide F precursor (2.02 FC; FDR rank 76), 
which encodes a neuropeptide that regulates food intake [43]; feeding is expected to increase as the females increase their egg-laying [44], and from our results this appears to be an early post-mating response.

\section{Gene expression in head tissues 24-hours post-mating}

Of the top 100 FDR ranked genes, 77 genes are repressed and 23 genes are induced 24 hours postmating (Table 4 and Additional file 2 for biological enrichment). Of these 100 genes, 59 repressed genes and 12 induced genes show at least two-fold differences in expression (Table 3 and Additional file 1).

The genes larval serum protein 2 (Lsp2) and Lsp1beta are two of the most highly repressed genes (Table 3 ), suggesting that a major shift in metabolic state is part of the post-mating response, as the products of these genes function as fat body nutrient reservoirs for the storage of amino acids [26,31]. In addition, CG8147, which is the gene that is the most highly induced in head tissues of mated females, also encodes a product involved in metabolic processes and has predicted alkaline phosphatase activity [31].

\section{Genes with lower expression in females 24 hours after mating}

Analyses of the gene ontologies of the 77 repressed genes supports the reduced expression of metabolic genes post-mating. Nearly all of the 115 biological process categories significantly overrepresented are metabolic categories (Additional file 1). Furthermore, 19 genes were also identified in studies examining gene expression after 24 hours of starvation [identified using Flymine $[33,45]]$. These analyses support the idea that in head tissues a shift in metabolic state, nutrient utilization, and storage is involved with the post-mating response as early as 24 hours postmating.

As validation of the experimental approach, 57 of the 77 genes repressed in head tissues at the 24-hour postmating stage have been identified as highly expressed in the adult head (identified using Flymine [33]), by studies that examined tissue-specific gene expression [46]. Sixty of the 77 genes were identified as expressed in the adult fat body, suggesting that they are also likely expressed in the head fat body in this study. Many of the 77 genes are expressed in both mated and virgin spermatheca female tissues ( 51 and 60 genes, respectively). This latter observation can be reconciled by the fact that both the head and spermatheca tissues either contain or are closely associated with fat body tissue. Genes that function in the nervous system were also identified, including 12 genes expressed in the brain and 11 genes expressed in the ventral nerve cord.
Table 4 The ten most significant GO categories for genes with significant expression differences at each time point

\begin{tabular}{lrr}
\hline Higher in mated females 0-2 hours post-mating & \\
\cline { 1 - 2 } GO Term & P value & $\begin{array}{r}\text { Number of } \\
\text { genes }\end{array}$ \\
dopamine metabolic process & $4.72 \mathrm{E}-04$ & 2 \\
anatomical structure development & $9.53 \mathrm{E}-04$ & 18 \\
developmental process & $1.26 \mathrm{E}-03$ & 20 \\
catecholamine metabolic process & $2.04 \mathrm{E}-03$ & 2 \\
catechol metabolic process & $2.05 \mathrm{E}-03$ & 2 \\
phenol metabolic process & $2.05 \mathrm{E}-03$ & 2 \\
diol metabolic process & $2.05 \mathrm{E}-03$ & 2 \\
multicellular organismal process & $2.29 \mathrm{E}-03$ & 22 \\
system development & $3.08 \mathrm{E}-03$ & 15 \\
positive regulation of multicellular organism & $3.69 \mathrm{E}-03$ & 2
\end{tabular}
growth

Lower in mated females $0-2$ hours post-mating

GO Term

$P$ value

Number of genes

response to abiotic stimulus

2.18E-05

response to heat

9.81E-04

$1.44 \mathrm{E}-03$

$1.66 \mathrm{E}-03$

alcohol metabolic process

$3.17 \mathrm{E}-03$

steroid catabolic process

$3.18 \mathrm{E}-03$

cholesterol catabolic process

$3.19 \mathrm{E}-03$

prenol metabolic process

$3.20 \mathrm{E}-03$

prenol biosynthetic process

3.20E-03

polyprenol metabolic process

$3.21 \mathrm{E}-03$

polyprenol biosynthetic process

Higher in mated females 24 hours post-mating

GO Term response to light stimulus

$P$ value

response to radiation

5.95E-04

absorption of visible light

9.19E-04

$1.98 \mathrm{E}-03$

arginine catabolic process to glutamate

positive regulation of biosynthetic process

$1.98 \mathrm{E}-03$

$2.68 \mathrm{E}-03$

positive regulation of cellular biosynthetic

$2.68 \mathrm{E}-03$

process

visual perception

2.86E-03

sensory perception of light stimulus

$3.01 \mathrm{E}-03$

positive regulation of cellular metabolic

3.33E-03 process

positive regulation of metabolic process $\quad 3.47 \mathrm{E}-03$

Lower in mated females 24 hours post-mating

GO Term

$P$ value

Number of genes

small molecule metabolic process

3.61E-12

$6.94 \mathrm{E}-11$

26

organic acid metabolic process

$6.95 \mathrm{E}-11$

6.95E-11

2.16E-10

$1.24 \mathrm{E}-07$

$1.24 \mathrm{E}-07$

$1.24 \mathrm{E}-07$

Number of genes

3

3

1

cellular ketone metabolic process

pentose-phosphate shunt

NADP metabolic process

NADPH regeneration 
Table 4: The ten most significant GO categories for genes with significant expression differences at each time point (Continued)

\begin{tabular}{|c|c|c|}
\hline nicotinamide metabolic process & 7.35E-07 & 4 \\
\hline alkaloid metabolic process & 7.37E-07 & \\
\hline \multicolumn{3}{|c|}{ Higher in mated females 48 hours post-mating } \\
\hline GO Term & $P$ value & $\begin{array}{r}\text { Number of } \\
\text { genes }\end{array}$ \\
\hline phosphatidylserine metabolic process & 2.23E-03 & 1 \\
\hline phosphatidylserine biosynthetic process & 2.24E-03 & 1 \\
\hline male courtship behavior & 2.25E-03 & 1 \\
\hline arginine catabolic process to glutamate & 2.25E-03 & 1 \\
\hline D-ribose metabolic process & 4.55E-03 & 1 \\
\hline arginine catabolic process & 4.57E-03 & 1 \\
\hline mating behavior, sex discrimination & 4.57E-03 & 1 \\
\hline cellular process & $9.92 \mathrm{E}-01$ & 8 \\
\hline \multicolumn{3}{|c|}{$\underline{\text { Lower in mated females } 48 \text { hours post-mating }}$} \\
\hline GO Term & $P$ value & $\begin{array}{r}\text { Number of } \\
\text { genes }\end{array}$ \\
\hline small molecule metabolic process & $1.40 \mathrm{E}-12$ & 26 \\
\hline organic acid metabolic process & 4.52E-10 & 15 \\
\hline carboxylic acid metabolic process & 4.54E-10 & 15 \\
\hline oxoacid metabolic process & 4.54E-10 & 15 \\
\hline cellular ketone metabolic process & $1.31 \mathrm{E}-09$ & 15 \\
\hline hexose metabolic process & 7.43E-09 & 9 \\
\hline monosaccharide metabolic process & $2.86 \mathrm{E}-08$ & 9 \\
\hline pentose-phosphate shunt & 1.10E-07 & 4 \\
\hline NADP metabolic process & 1.10E-07 & 4 \\
\hline $\mathrm{NADPH}$ regeneration & 1.10E-07 & 4 \\
\hline \multicolumn{3}{|c|}{ Higher in mated females 72 hours post-mating } \\
\hline GO Term & $P$ value & $\begin{array}{r}\text { Number of } \\
\text { genes }\end{array}$ \\
\hline phosphatidylserine metabolic process & $3.14 \mathrm{E}-03$ & 1 \\
\hline phosphatidylserine biosynthetic process & $3.14 \mathrm{E}-03$ & 1 \\
\hline leucyl-tRNA aminoacylation & $6.34 \mathrm{E}-03$ & 1 \\
\hline asparagine metabolic process & 6.37E-03 & 1 \\
\hline asparagine biosynthetic process & 6.37E-03 & 1 \\
\hline organic acid metabolic process & 7.63E-03 & 4 \\
\hline carboxylic acid metabolic process & 7.67E-03 & 4 \\
\hline oxoacid metabolic process & 7.67E-03 & 4 \\
\hline biological regulation & 9.97E-01 & 2 \\
\hline \multicolumn{3}{|c|}{ Lower in mated females 72 hours post-mating } \\
\hline GO Term & $P$ value & $\begin{array}{r}\text { Number of } \\
\text { genes }\end{array}$ \\
\hline oxidation reduction & 8.05E-06 & 13 \\
\hline organic acid metabolic process & 3.75E-05 & 9 \\
\hline carboxylic acid metabolic process & 3.76E-05 & 9 \\
\hline oxoacid metabolic process & $3.76 \mathrm{E}-05$ & 9 \\
\hline aspartate metabolic process & 4.36E-05 & 2 \\
\hline cellular ketone metabolic process & $6.82 \mathrm{E}-05$ & 9 \\
\hline purine base biosynthetic process & 2.61E-04 & 2 \\
\hline glutamate biosynthetic process & 4.35E-04 & 2 \\
\hline small molecule metabolic process & 5.26E-04 & 13 \\
\hline 'de novo' IMP biosynthetic process & $6.53 \mathrm{E}-04$ & 2 \\
\hline
\end{tabular}

\section{Genes with higher expression in females 24 hours after} mating

Surprisingly, of the 23 genes with higher expression in mated females, 11 genes have enriched expression in the adult eye [46], or are known to function in visual transduction (include rhodopsin 4, rhodopsin 5, retinin and trp-like). This suggests that changes in the visual transduction system may accompany aspects of the post-mating response, which is an unexpected observation. It is also possible that the products of these genes have roles in other head tissues and that the change in their expression may not affect visual transduction, but rather, other physiological processes. Finally, the increased expression of these genes may reflect a change post-mating in the relative abundance of eye tissue, as compared to other head tissues, like the fat body. Our observation that there is a substantial repression of gene expression in the fat body post-mating may reflect that there is a decrease in fat body tissue in the head postmating. Given the way microarray data are normalized, this could result in an apparent increase in expression of genes in other tissues, like the eye.

\section{Gene expression in head tissues 48-hours post-mating}

Of the top 100 FDR ranked genes, 75 genes are repressed and 25 genes are induced 48 hours postmating (Table 4 and Additional file 1 and 2). Of these 100 genes, 39 repressed and 14 induced genes show at least two-fold differences in expression (Table 3 and Additional file 1 and 2).

The genes Lsp2 and Lsp1beta, which encode nutrient reservoirs, are again among the most strongly repressed genes in head tissues of mated females (Table 3), as are takeout (to; 2.86 FC; FDR rank 7), and Odorant binding proteins 99a (Obp99a; 4.02 FC; FDR rank 9) and Obp99b (9.09 FC; FDR rank 17; Table 3).

to expression is also repressed in adult head tissues in response to mating at 24 and 72 hours post-mating. This result is consistent with the previous finding that the to encoded product, which is highly expressed in the adult fat body, is more abundant in the hemolymph of males than in females [25]. to has previously been shown to function in the behavioral response to starvation, adult feeding behavior, male courtship behavior, and to expression is under circadian regulation [25,47-51]. The results presented here suggest another behavioral and physiological role for to in the female post-mating response.

In contrast to the results presented here, Obp99a was found to be more highly expressed in mated females at two time points (1-3 hours and 12 hours post-mating; [19]), when the whole animal was assayed, supporting the idea that different tissues have different gene expression responses post-mating. It has been shown in one 
study that $O b p 99 b$ has substantial male-biased expression in head tissues [39], however in our previous study we observed no significant sex-biased expression in head tissues [24]. Also, Obp99b is up-regulated in males that have courted females [29], is higher in fruitless mutant males that display very reduced courtship behaviors (2.56 FC; FDR rank 4; [24]), and is down-regulated in males that have been starved [32]. In females, ectopic expression of Obp99b leads to reduced virgin female receptivity and copulation frequency [39]. Taken together, this suggests that expression of $O b p 99 b$ is sensitive to and perhaps influences nutrient status and reproductive status in both males and females.

\section{Genes with lower expression in females $\mathbf{4 8}$ hours after mating}

Of the 75 genes that are repressed 48 hours after mating, 44 were also repressed 24 hours after mating and include many of the genes involved in metabolism [identified using Flymine [33]]. The remaining 31 repressed genes at 48 hours, but not at 24 hours, are enriched with genes whose products are involved in glycogen and glucan metabolism and biosynthesis $(p<$ 0.0001 , two genes). This suggests that the timing of aspects of the metabolic shift observed post-mating depends on the biosynthetic pathway. Additional repressed genes in mated females at 48 but not at 24 hours include Obp99a and Neuropeptide-like precursor 3 (Nplp3; 2.13 FC; FDR rank 49), which in contrast to this study, was induced post-mating when whole animal tissues were examined [19]

\section{Genes with higher expression in females $\mathbf{4 8}$ hours after mating}

Of the 25 genes induced in mated head tissues at 48 hours, six were also induced at 24 hours and include genes encoding metabolic enzymes. The 19 remaining genes include seven genes that are expressed in the nervous system (fruitless, Regulator of G-protein signaling 7 , out at first, CG8745, Crk, CG11425, and CG11347), as assessed using Flyatlas data [46]. Overall, the gene expression changes observed at 24- and 48-hours postmating are similar, however, more genes with known expression in the CNS are induced at 48 hours.

\section{Gene expression in head tissues 72-hours post-mating} Of the top 100 FDR ranked genes, 69 genes are repressed and 31 genes are induced 72 hours post-mating (Table 4 and Additional file 1 and 2). Of these 100 genes, 18 repressed and 8 induced genes show at least two-fold differences in expression (Table 3 and Additional file 1). The observation that there are fewer genes with robust differences in expression at 72 hours (FC $>2$ ), as compared to 24 and 48 hours, suggests that mating-induced transcriptional changes begin to diminish in magnitude after 48 hours.

The genes $L s p 2$, to, Obp $99 a$, and $O b p 99 b$ are again among the most repressed genes, as they were at 24 hours (Table 3). CG3348 is the most highly induced gene at 72 hours in mated head tissue ( six-fold change) and was among the most highly induced genes at 24- and 48-hours post-mating. CG3348 encodes a product predicted to be involved in chitin metabolism [31], which suggests that changes in the regulation of cuticle synthesis underlie aspects of the female postmating response, perhaps including the cuticle that forms the head capsule or the cuticle that is part of the adult trachea, the fly respiratory system. CG3348 was also shown to have greater than two-fold up-regulated expression in mated females, which is induced by sperm transfer in the absence of Acps [20].

\section{Genes with lower expression in females 72 hours after mating}

Of the 69 genes that were repressed 72 hours post-mating, 19 were also repressed at 48 hours post-mating and include genes involved in metabolism. The remaining 50 genes that are repressed post-mating are enriched for those involved in immune response function (antibacterial humoral response; $p<0.0008$; three genes; Additional file 1). Several genes involved in lipid metabolism are repressed at this stage (brummer, doppelganger von brummer, secretory Phospholipase A2). The observation that Insulin-like peptide 5 (1.51 FC; FDR rank 62) is in this gene set suggests that the metabolic state of head tissues is transmitted systemically to elicit responses in different tissues, as has been shown for Insulin like peptides produced in the head that influence egg production [52].

\section{Genes with higher expression in females 72 hours after mating}

Of the 31 genes induced 72 hours post-mating, eight were also more abundant at 48 hours. The remaining 23 include sugarbabe (1.49 FC; FDR rank 42), a zinc-finger transcription factor that is induced in fat body and gut tissues in response to sugar [45], suggesting that sugar levels are higher in head tissues at 72-hours post-mating than at earlier times [45].

\section{Gene expression in dissected brain tissue 24-hours post- mating}

To more specifically identify genes that play a role in the CNS to mediate post-mating behavioral changes, gene expression analyses were performed on dissected brain tissues 24 hours post-mating. Of the top 100 FDR ranked genes, 28 genes are repressed and 72 genes are induced in brain tissue 24 hours after mating. Of these 
100 genes, five repressed and 20 induced genes show at least two-fold differences in expression (Table 2 and Additional file 1).

\section{Genes with lower expression in CNS tissues of females 24 hours after mating}

Of the 28 genes that are repressed in dissected brain tissues 24 hours post-mating, only one gene, Obp99b, was also identified as repressed in whole head tissues 24 hours post-mating. This suggests that microarray experiments performed with RNA from whole head tissues lack the sensitivity to detect many gene expression changes in brain tissues. Twenty-two of the 28 genes have previously been shown to be expressed in brain tissues [46], validating the experimental approach. shaker, a voltage-gated cation channel, is among the genes that show the most substantial repression post-mating (2 FC; FDR rank 45). Five of the 28 genes have ion channel activity ( $\mathrm{pHCl}$, shaker, cacophony, $\mathrm{Ca}^{2+}$-channel-proteinbeta-subunit, tipE homolog 1), suggesting that regulation of these genes is involved in the post-mating behavioral response. In addition, four of the 28 genes encode products with mRNA binding activity (bruno-3, hephaestus, orb2, RNA-binding protein 6), two encode products with axon guidance activity (smooth, Protein tyrosine phosphatase 99A) and one encodes a product with neurotransmitter transport activity (complexin) [31]. A set of critical neurons that respond to SP are not in the brain $[12,13]$, but the gene classes identified here suggest that there are substantial changes in neurophysiology in the brain that may underlie post-mating behavioral changes.

\section{Genes with higher expression in CNS tissues of females 24 hours after mating}

Of the 72 genes that are up-regulated in dissected brain tissues 24 hours post-mating, five genes were also identified as induced in whole head tissues 24 hours after mating; these include retinin, which is expressed in the visual system, and four additional genes with various functions (chitin binding, alkaline phosphatase activity and two of unknown function). Additionally, several genes known to be expressed in brain tissues were identified, including those encoding proteins that function in chromatin assembly (dre4), mRNA localization (larsen) and wingless protein binding (nimrod B4), suggesting that several different molecular mechanisms underlie the post-mating response in the brain. Four genes in this set are also involved in immune function. SP is known to induce the innate immune response $[35,53]$, suggesting that SP or other factors can elicit an immune response in the brain.

Expression of mating-responsive genes in different tissues To determine how similar the post-mating response is in different tissues, we compared the 309 genes identified here as having post-mating gene expression changes in head tissues with genes identified in previous studies that examined different tissues or whole animals. Only four genes identified here (fit, CG6910, Lsp1beta, psd) were also identified as differentially expressed in mated versus virgin whole females [19]. Adult reproductive tissues may express a similar group of matingresponsive genes as head tissues, given that many genes identified here as expressed in head tissues are also expressed in spermathecae. Furthermore, eighteen genes identified here were previously identified as differentially expressed in mated and virgin female reproductive tissues (Additional file 3) [22]. In both comparisons noted, whether the gene is induced or repressed varies with the tissue and time point examined.

To determine whether the genes identified here as mating-responsive in female head tissues are also sexdifferentially expressed, we compared genes identified in this study with our previous study that identified genes with sex-differential expression in head tissues [24]. Sixteen mating-responsive genes in females were also identified as having significant sex-differential expression. The sex-biased expression does not always conform to what might be expected; for example, a gene that is upregulated post-mating might be expected to have female-biased expression, but only about half the genes show this pattern (Additional file 3). Therefore, most genes that are induced post-mating at the four times examined here do not show sex-differential expression in adults that are $0-24$ hours old, which may also be due to differences in reproductive status and age between the two studies.

\section{Discussion}

We have identified hundreds of genes that change expression post-mating in female head and brain tissues. At the four time points examined, a wide range of genes showed significant differential expression between mated and virgin female head tissues [0-2 (237 genes), 24 (326 genes), 48 (449 genes), and 72 (545 genes) hours post-mating, $P<0.05]$. The largest functional group of significantly differentially expressed genes encodes products involved in metabolism that are expressed in the fat body. We observe that many of these genes are repressed starting at 24 hours post-mating, but not at the earlier $0-2$ hour post-mating time point. This is likely due to the fact that egg production is energetically costly and females shift from nutrient storage to utilization, as their stores are depleted. For example, Lsp1beta and Lsp2, which encode amino acid reservoirs [26,31], are among the most highly repressed genes at 24 and 48 hours post-mating, but not at the 0 2 hour post-mating stage. Given that many of the changes in expression affect genes that encode products 
involved in metabolism, it is not clear if this is a direct response to receipt of sperm and seminal fluid, or due to social interactions that occur during courtship and mating. It is likely that for many genes the expression changes are secondary responses to the dramatic changes in physiology that occur due to increased egg laying. Additional experiments that assay gene expression changes in females that mate with males that lack sperm or accessory gland proteins will help distinguish between these possibilities, as will assaying gene expression in females that do not produce eggs, and in females that interact with males, but do not copulate.

Furthermore, it is not clear how many of the changes in gene expression in the head direct the female postmating response, as opposed to being a consequence of changes in reproductive status; this is especially the case for genes with expression in head fat body. Other work has suggested that the head fat body may have additional roles beyond energetic support and might have complex interactions with the nervous system, ultimately influencing sex-specific behaviors. For example, a masculinized fat body is required for wild type levels of male courtship behavior [25]. Additionally, our previous work showed that fruitless mutations affecting male courtship behaviors also affect male gene expression in the head fat body [24]. Given that fruitless is expressed in the nervous system, this suggests that the physiology of the nervous system and/or the consequence of performing courtship behaviors influences gene expression in the fat body, which is supported by an additional study [29]. The idea that there are interactions between the fat body and the nervous system is also supported by the observation that silencing or activating the fruitless-expressing neurons that underlie sex-specific behaviors in both males and females can substantially shift fat content in adult flies [54]. Additionally, it has been shown that gene expression in the fat body affects female reproductive behaviors, with ectopic expression of $O b p 99 b$ in female fat body tissues leading to reduced receptivity and mating success [39]. Taken together, one hypothesis is that there is a dynamic interplay between head fat body metabolic state and endocrine function and nervous system function that ultimately influence and direct adult reproductive behavior and physiology.

Gene expression changes in the brain are likely to mediate some of the long-term behavioral changes that occur post-mating, which lasts about one week and includes reduced receptivity to mating and increased egg laying. While much progress has been made in understanding at a molecular-genetic level how SP, SPR and other Acps influence the post-mating behavioral response [reviewed in $[1,2]]$, most of the downstream effectors in the brain are not known, especially those that mediate the long-term behavioral female post-mating response. In this study, several genes with expression in the brain that encode products that function in neurophysiology were identified, with five having ion channel activities with known roles in behaviors [31]. An important next step will be to determine where these channels are expressed in females and how changes in their expression levels influence neurophysiology and behavior. To identify additional genes that mediate the post-mating response in brain tissue, it will also be important to assay gene expression in the nervous system at more time points, using more sensitive techniques, such as deep-sequencing technologies.

Many of the genes identified in this study with significant differential expression in head tissues were also identified as significantly enriched in gene lists from other expression studies (Benjamini and Hochberg $P<$ 0.01; [assessed using Flymine [33]]), including those assaying circadian regulation of gene expression $(22$ genes [50]; 31 genes [55]), gene expression in response to nutrient status (15 genes [32], 17 genes [45]), sexdifferential gene expression (9 genes [24]), and postmating gene expression in whole animals (13 genes [19]). This overlap demonstrates that in the future it will be important to use an integrated, systems-level approach to understand this complex behavior.

\section{Conclusions}

Substantial changes occur in the expression of genes in female head tissues after mating. These results provide new insights into the physiological and metabolic changes that accompany changes in female behaviors. By identifying the gene expression changes prompted by mating, this dataset provides a basis for future mechanistic studies that examine how specific genes mediate behavioral and physiological changes in Drosophila females post-mating. Additionally, understanding how these changes in gene expression orchestrate the postmating response in Drosophila may provide insight into the reproductive behavior of more complex animals.

\section{Methods}

\section{Drosophila stocks and husbandry}

Flies were raised at $25^{\circ} \mathrm{C}$ under a 12-hour light and 12hour dark cycle, on standard cornmeal food media. In all experiments, female flies were the wild-type Canton $\mathrm{S}$ strain. The male flies were the genotype $w ; P[U A S-d j-$ $G F P]$, which contains a transgene that expresses the Don Juan protein tagged with GFP (DJ-GFP) [56]. DJGFP associates with the sperm tail allowing visual confirmation of mating by the presence of GFP in the reproductive tract of mated females. 


\section{Mating conditions and fly collections}

Virgin Canton S females and w; P[UAS-dj-GFP] males were collected within five to eight hours of eclosion and aged for five days. On the fifth day, females and males were combined (ten females with 20 males) for two hours between the time period of 6-8 hours after the lights came on in the incubator. Females were then sorted under $\mathrm{CO}_{2}$ anesthesia using a fluorescence microscope. Only females with strong GFP expression in the lower abdomen, indicating the presence of sperm in the reproductive tract, were collected and aged on food. The mated females were then collected 24, 48 or 72 hours post-mating by tapping them without anesthesia into a cryovial, and immediately snap-freezing the flies in liquid nitrogen. Age matched virgin females were collected in a similar manner. For the 02 hour time point, animals were anaesthetized after the two hour mating period, identified as mated females and were immediately snap frozen in liquid nitrogen. The virgin females collected for the 0-2 hour time point were not exposed to males, but were anaesthetized to control for gene expression differences due to $\mathrm{CO}_{2}$ exposure, and then snap frozen. For experiments analyzing gene expression in dissected brain tissues, flies were mated as above and 24 hours after mating were lightly anaesthetized individually on ice. Brain tissue was dissected quickly in icecold $1 \times$ phosphate buffered saline (PBS) made with RNase-free water. The brain tissue was rinsed in $1 \times$ PBS to remove non-brain tissue and immediately placed in icecold Trizol (Invitrogen) until RNA extraction.

\section{RNA extraction and microarray experiments}

The two-color, glass-slide microarray platform, labeled probe preparation and hybridization conditions were performed as previously described [27]. Adult heads were snapped-off from the body by shaking the frozen flies in the cryovial. The frozen heads were sorted from the bodies on plastic cooled on dry ice. Total RNA was extracted from $\sim 100$ heads per sample, using Trizol (Invitrogen). All experiments were performed with four independent biological samples, and a dye swap design to control for biases in dye incorporation. For experiments using RNA derived from head tissue, $20 \mu \mathrm{g}$ of total RNA was used to generate labeled cDNA. For experiments using dissected brain tissue, 20 brains were dissected per sample and cRNA probes were made by converting the RNA to double stranded cDNA that is flanked by a T7 promoter. T7 RNA polymerase was then used to generate cRNA, using the Amino Allyl MessageAmp II aRNA Amplification Kit (Ambion).

\section{Microarray Data Statistical Analyses}

Statistical analyses were performed as previously described [27]. To identify genes with significant differences in gene expression, a False Discovery Rate (FDR) method was employed. First $P$ values were determined using the LIMMA package of BioConductor in the program $\mathrm{R}$ and converted to FDR values [57-59]. A hypergeometric statistical test was used to determine the significance of enrichment of functional groups, with the Benjamini and Hochberg multiple hypothesis test correction at a $p<0.01$ threshold, implemented using Flymine [33]. For comparisons with the McGraw et al. 2008 study, analyses were limited to genes from that study that showed $>$ two-fold difference [19]. For comparisons with the Goldman and Arbeitman study, the analyses were limited to the top 100 FDR ranked genes with sex-differential expression in the same Canton S strain used here [24]. All data can be downloaded from the GEO website: GSE22390.

\section{Ethics statement}

The research performed in this study on the fruit fly, Drosophila melanogaster, did not need to be approved by an ethics committee.

\section{Additional material}

\section{Additional file 1: FDR 100 and GO 309 genes. The top FDR ranked genes with gene expression changes at $0-2,24,48$ and 72 hours post- mating. The top FDR ranked genes with gene expression changes in brain tissues 24 hours post-mating. Gene Ontology functional enrichment of 309 genes that are the intersection of genes identified in head tissues at four time points assayed. \\ Additional file 2: GO for each list. GO analyses of lists of top 100 FDR ranked genes from Additional file 1.}

Additional file 3: Comparisons to other datasets. Analysis of overlap between this study and Mack et al study and analyses of overlap between this study and Goldman et al study.

\section{Abbreviations}

(SP): Sex peptide; (Acps): Accessory Gland Proteins; (Lsp): Larval serum protein; (CNS): Central Nervous System; (Obp): Odorant Binding Protein; (FDR): False Discovery Rate; (GO): Gene Ontology; (fit): female-independent-oftransformer; (to): takeout; (GO): Gene Ontology.

\section{Acknowledgements}

The work was funded by NIH grant 1R01GM073039 awarded to MNA; we are grateful for the support. We thank Oscar Aparicio for helpful comments on the manuscript. This manuscript is dedicated to the memory of a good friend and fellow Drosophila researcher, John Sisson.

\section{Authors' contributions}

All of the authors contributed either to the data generation, data analysis, reagents, or paper writing. AN and ES performed the microarray experiments. ML performed the statistical analyses. SK generated the Venn Diagrams. All authors contributed to the analyses and writing and approved the final manuscript.

Received: 22 June 2010 Accepted: 6 October 2010 Published: 6 October 2010

\section{References}

1. Wolfner MF: The gifts that keep on giving: physiological functions and evolutionary dynamics of male seminal proteins in Drosophila. Heredity 2002, 88(2):85-93. 
2. Kubli E: Sex-peptides: seminal peptides of the Drosophila male. Cell Mol Life Sci 2003, 60(8):1689-1704.

3. Wolfner MF: Battle and ballet: molecular interactions between the sexes in Drosophila. J Hered 2009, 100(4):399-410.

4. Chen PS, Stumm-Zollinger E, Aigaki T, Balmer J, Bienz M, Bohlen P: A male accessory gland peptide that regulates reproductive behavior of female D. melanogaster. Cell 1988, 54(3):291-298.

5. Aigaki T, Fleischmann I, Chen PS, Kubli E: Ectopic expression of sex peptide alters reproductive behavior of female D. melanogaster. Neuron 1991, 7(4):557-563

6. Chapman T, Bangham J, Vinti G, Seifried B, Lung O, Wolfner MF, Smith HK, Partridge $L$ : The sex peptide of Drosophila melanogaster: female postmating responses analyzed by using RNA interference. Proc Natl Acad Sci USA 2003, 100(17):9923-9928.

7. Liu H, Kubli E: Sex-peptide is the molecular basis of the sperm effect in Drosophila melanogaster. Proc Natl Acad Sci USA 2003, 100(17):9929-9933.

8. Pilpel N, Nezer I, Applebaum SW, Heifetz Y: Mating-increases trypsin in female Drosophila hemolymph. Insect Biochem Mol Biol 2008, 38(3):320-330

9. Peng J, Chen S, Busser S, Liu H, Honegger T, Kubli E: Gradual release of sperm bound sex-peptide controls female postmating behavior in Drosophila. Curr Biol 2005, 15(3):207-213.

10. Ram KR, Wolfner MF: Sustained post-mating response in Drosophila melanogaster requires multiple seminal fluid proteins. PLOS Genet 2007, 3(12):e238.

11. Yapici N, Kim YJ, Ribeiro C, Dickson BJ: A receptor that mediates the postmating switch in Drosophila reproductive behaviour. Nature 2008, 451(7174):33-37.

12. Hasemeyer M, Yapici N, Heberlein U, Dickson BJ: Sensory neurons in the Drosophila genital tract regulate female reproductive behavior. Neuron 2009, 61(4):511-518.

13. Yang $\mathrm{CH}$, Rumpf $\mathrm{S}$, Xiang $\mathrm{Y}$, Gordon $\mathrm{MD}$, Song $\mathrm{W}$, Jan $\mathrm{LY}$, Jan $\mathrm{YN}$ : Control of the postmating behavioral switch in Drosophila females by internal sensory neurons. Neuron 2009, 61(4):519-526.

14. Ribeiro C, Dickson BJ: Sex peptide receptor and neuronal TOR/S6K signaling modulate nutrient balancing in Drosophila. Curr Biol 20(11):1000-1005.

15. Vargas MA, Luo N, Yamaguchi A, Kapahi P: A role for S6 kinase and serotonin in postmating dietary switch and balance of nutrients in $\mathrm{D}$. melanogaster. Curr Biol 20(11):1006-1011.

16. Carvalho GB, Kapahi $P$, Anderson DJ, Benzer S: Allocrine modulation of feeding behavior by the Sex Peptide of Drosophila. Curr Biol 2006, 16(7):692-696.

17. Heifetz $Y$, Lung $O$, Frongillo EA Jr, Wolfner MF: The Drosophila seminal fluid protein Acp26Aa stimulates release of oocytes by the ovary. Curr Biol 2000, 10(2):99-102.

18. Neubaum DM, Wolfner MF: Mated Drosophila melanogaster females require a seminal fluid protein, Acp36DE, to store sperm efficiently. Genetics 1999, 153(2):845-857.

19. McGraw LA, Clark AG, Wolfner MF: Post-mating gene expression profiles of female Drosophila melanogaster in response to time and to four male accessory gland proteins. Genetics 2008, 179(3):1395-1408.

20. McGraw LA, Gibson G, Clark AG, Wolfner MF: Genes regulated by mating sperm, or seminal proteins in mated female Drosophila melanogaster. Curr Biol 2004, 14(16):1509-1514.

21. Lawniczak MK, Begun DJ: A genome-wide analysis of courting and mating responses in Drosophila melanogaster females. Genome 2004, 47(5):900-910

22. Mack PD, Kapelnikov A, Heifetz Y, Bender M: Mating-responsive genes in reproductive tissues of female Drosophila melanogaster. Proc Natl Acad Sci USA 2006, 103(27):10358-10363.

23. Arbeitman MN, Fleming AA, Siegal ML, Null BH, Baker BS: A genomic analysis of Drosophila somatic sexual differentiation and its regulation. Development 2004, 131(9):2007-2021.

24. Goldman TD, Arbeitman MN: Genomic and functional studies of Drosophila sex hierarchy regulated gene expression in adult head and nervous system tissues. PLoS Genet 2007, 3(11):e216

25. Lazareva AA, Roman G, Mattox W, Hardin PE, Dauwalder B: A role for the adult fat body in Drosophila male courtship behavior. PLoS Genet 2007, 3(1):e16.
26. Liu Y, Liu H, Liu S, Wang S, Jiang RJ, Li S: Hormonal and nutritional regulation of insect fat body development and function. Arch Insect Biochem Physiol 2009, 71(1):16-30.

27. Lebo MS, Sanders LE, Sun F, Arbeitman MN: Somatic, germline and sex hierarchy regulated gene expression during Drosophila metamorphosis. BMC Genomics 2009, 10:80

28. Arbeitman MN, Furlong EE, Imam F, Johnson E, Null BH, Baker BS, Krasnow MA, Scott MP, Davis RW, White KP: Gene expression during the life cycle of Drosophila melanogaster. Science 2002, 297(5590):2270-2275.

29. Carney GE: A rapid genome-wide response to Drosophila melanogaster social interactions. BMC Genomics 2007, 8:288.

30. Parisi MJ, Gupta V, Sturgill D, Warren JT, Jallon JM, Malone JH, Zhang Y, Gilbert LI, Oliver B: Germline-dependent gene expression in distant nongonadal somatic tissues of Drosophila. BMC Genomics 11(1):346.

31. Tweedie S, Ashburner M, Falls K, Leyland P, McQuilton P, Marygold S, Millburn G, Osumi-Sutherland D, Schroeder A, Seal R, et al: FlyBase: enhancing Drosophila Gene Ontology annotations. Nucleic Acids Res 2009, 37 Database: D555-559.

32. Fujikawa K, Takahashi A, Nishimura A, Itoh M, Takano-Shimizu T, Ozaki M: Characteristics of genes up-regulated and down-regulated after $24 \mathrm{~h}$ starvation in the head of Drosophila. Gene 2009, 446(1):11-17.

33. Lyne R, Smith R, Rutherford K, Wakeling M, Varley A, Guillier F, Janssens $H$, Ji W, McLaren P, North P, et al: FlyMine: an integrated database for Drosophila and Anopheles genomics. Genome Biol 2007, 8(7):R129.

34. Ding Z, Haussmann I, Ottiger M, Kubli E: Sex-peptides bind to two molecularly different targets in Drosophila melanogaster females. $J$ Neurobiol 2003, 55(3):372-384

35. Peng J, Zipperlen P, Kubli E: Drosophila sex-peptide stimulates female innate immune system after mating via the Toll and Imd pathways. Curr Biol 2005, 15(18):1690-1694.

36. Neckameyer WS: Dopamine modulates female sexual receptivity in Drosophila melanogaster. J Neurogenet 1998, 12(2):101-114.

37. Hintermann E, Grieder NC, Amherd R, Brodbeck D, Meyer UA: Cloning of an arylalkylamine $\mathrm{N}$-acetyltransferase (aaNAT1) from Drosophila melanogaster expressed in the nervous system and the gut. Proc Natl Acad Sci USA 1996, 93(22):12315-12320.

38. Brodbeck D, Amherd R, Callaerts P, Hintermann E, Meyer UA, Affolter M: Molecular and biochemical characterization of the aaNAT1 (Dat) locus in Drosophila melanogaster: differential expression of two gene products. DNA Cell Biol 1998, 17(7):621-633.

39. Fujii $\mathrm{S}$, Amrein $\mathrm{H}$ : Genes expressed in the Drosophila head reveal a role for fat cells in sex-specific physiology. EMBO J 2002, 21(20):5353-5363.

40. Johnston LA, Prober DA, Edgar BA, Eisenman RN, Gallant P: Drosophila myc regulates cellular growth during development. Cell 1999, 98(6):779-790.

41. Ryner LC, Goodwin SF, Castrillon DH, Anand A, Villella A, Baker BS, Hall JC, Taylor BJ, Wasserman SA: Control of male sexual behavior and sexual orientation in Drosophila by the fruitless gene. Cell 1996, 87(6):1079-1089.

42. Lee G, Foss M, Goodwin SF, Carlo T, Taylor BJ, Hall JC: Spatial, temporal, and sexually dimorphic expression patterns of the fruitless gene in the Drosophila central nervous system. J Neurobiol 2000, 43(4):404-426.

43. Lee KS, You KH, Choo JK, Han YM, Yu K: Drosophila short neuropeptide F regulates food intake and body size. J Biol Chem 2004, 279(49):50781-50789.

44. Barnes Al, Wigby S, Boone JM, Partridge $L$, Chapman T: Feeding, fecundity and lifespan in female Drosophila melanogaster. Proc Biol Sci 2008, 275(1643):1675-1683

45. Zinke I, Schutz CS, Katzenberger JD, Bauer M, Pankratz MJ: Nutrient control of gene expression in Drosophila: microarray analysis of starvation and sugar-dependent response. EMBO J 2002, 21(22):6162-6173.

46. Chintapalli VR, Wang J, Dow JA: Using FlyAtlas to identify better Drosophila melanogaster models of human disease. Nat Genet 2007, 39(6):715-720.

47. So WV, Sarov-Blat L, Kotarski CK, McDonald MJ, Allada R, Rosbash M: takeout, a novel Drosophila gene under circadian clock transcriptional regulation. Mol Cell Biol 2000, 20(18):6935-6944.

48. Sarov-Blat L, So WV, Liu L, Rosbash M: The Drosophila takeout gene is a novel molecular link between circadian rhythms and feeding behavior. Cell 2000, 101(6):647-656.

49. Dauwalder B, Tsujimoto S, Moss J, Mattox W: The Drosophila takeout gene is regulated by the somatic sex-determination pathway and affects male courtship behavior. Genes Dev 2002, 16(22):2879-2892. 
50. McDonald MJ, Rosbash M: Microarray analysis and organization of circadian gene expression in Drosophila. Cell 2001, 107(5):567-578,

51. Claridge-Chang A, Wijnen H, Naef F, Boothroyd C, Rajewsky N, Young MW: Circadian regulation of gene expression systems in the Drosophila head. Neuron 2001, 32(4):657-671.

52. Drummond-Barbosa D, Spradling AC: Stem cells and their progeny respond to nutritional changes during Drosophila oogenesis. Dev Biol 2001, 231(1):265-278

53. Domanitskaya EV, Liu H, Chen S, Kubli E: The hydroxyproline motif of male sex peptide elicits the innate immune response in Drosophila females. FEBS J 2007, 274(21):5659-5668.

54. Al-Anzi B, Sapin V, Waters C, Zinn K, Wyman RJ, Benzer S: Obesity-blocking neurons in Drosophila. Neuron 2009, 63(3):329-341.

55. Ceriani MF, Hogenesch JB, Yanovsky M, Panda S, Straume M, Kay SA: Genome-wide expression analysis in Drosophila reveals genes controlling circadian behavior. J Neurosci 2002, 22(21):9305-9319.

56. Santel A, Winhauer T, Blumer N, Renkawitz-Pohl R: The Drosophila don juan (dj) gene encodes a novel sperm specific protein component characterized by an unusual domain of a repetitive amino acid motif. Mech Dev 1997, 64(1-2):19-30

57. Storey JD: The positive false discovery rate: A Bayesian interpretation and the q-value. Annals of Statistics 2003, 31: 2013-2035.

58. Smyth GK: Limma: linear models for microarray data. In Bioinformatics and Computational Biology Solutions using $R$ and Bioconductor. Edited by: $R$ Gentleman VC, Dudoit S, Irizarry R, Huber W. New York: Springer; 2005:397-420.

59. Smyth GK: Linear models and empirical bayes methods for assessing differential expression in microarray experiments. Statistical applications in genetics and molecular biology 2004, 3:Article 3.

doi:10.1186/1471-2164-11-541

Cite this article as: Dalton et al: Dynamic, mating-induced gene expression changes in female head and brain tissues of Drosophila melanogaster. BMC Genomics 2010 11:541.

\section{Submit your next manuscript to BioMed Central and take full advantage of:}

- Convenient online submission

- Thorough peer review

- No space constraints or color figure charges

- Immediate publication on acceptance

- Inclusion in PubMed, CAS, Scopus and Google Scholar

- Research which is freely available for redistribution

Submit your manuscript at www.biomedcentral.com/submit
Biomed Central 